\title{
In Situ Bioremediation of Crude Petroleum Oil Polluted Soil Using Mathematical Experimentation
}

\author{
Modupe Elizabeth Ojewumi, Moses Eterigho Emetere, \\ Damilola Elizabeth Babatunde, and Joshua Olusegun Okeniyi \\ Covenant University, Km 10, Idiroko Road, Canaan Land, PMB 1023, Sango Ota, Ogun State, Nigeria \\ Correspondence should be addressed to Modupe Elizabeth Ojewumi; modupe.ojewumi@covenantuniversity.edu.ng
}

Received 14 January 2017; Accepted 22 March 2017; Published 4 July 2017

Academic Editor: Justo Lobato

Copyright ( 2017 Modupe Elizabeth Ojewumi et al. This is an open access article distributed under the Creative Commons Attribution License, which permits unrestricted use, distribution, and reproduction in any medium, provided the original work is properly cited.

\begin{abstract}
Mathematical modelling of in situ (on site) bioremediation of crude petroleum polluted soil was investigated. An unsteady state mathematical model based on bulk flow of oil through the soil and molecular diffusion through the pores of the soil was developed. The parabolic partial differential equation developed was resolved into a system of ordinary differential equations (ODEs) by orthogonal collocation method and the necessary boundary condition was used. The resultant system of ODE was solved using fourth-order Runge-Kutta method. The simulated data gave a good agreement with experimental data.
\end{abstract}

\section{Introduction}

One of the major concerns of oil industry today is how to improve the recovery of large percentage of oil remaining unrecovered in the old and new depleted producing fields. Microbial treatment of oil well system and reservoir to control paraffin deposition is one of the efficient enhanced oil recovery methods [1]. Bioremediation project in practical sense is very expensive to execute. The accepted disposal methods of incineration or burial of insecure landfills are now expensive when amounts of contaminants are large. Mechanical and chemical methods generally used to remove hydrocarbons from contaminated sites have limited effectiveness and can be expensive [2]. The aftermath of bioremediation activities may be dangerous to life in the predetermined region. Hence, the first step towards achieving a safe bioremediation process is developing mathematical models that comprise all necessary parameters that determine the entire process.

References [3,4] reported that, in Taiwan, over 400 gas stations had encountered problems of contamination from petroleum hydrocarbons, due to aged pipelines. Leaks and accidental spills occur regularly during the exploration, production, refining, transport, and storage of petroleum and petroleum products [3]. Release of hydrocarbons into the environment whether accidentally or due to human activities is a main cause of water and soil pollution [4]. This is one of the major environmental problems today resulting from the activities of the petrochemical industry. Hydrocarbon components have been known to belong to the family of carcinogens and potent immunotoxic organic pollutants which are harmful to all living things both terrestrial and aquatic [5]. Soil contamination with hydrocarbons causes extensive damage of local system since accumulation of pollutants in animals and plant tissue may cause death or mutations [6]. Among the many techniques employed to decontaminate the affected sites, in situ bioremediation using indigenous microorganisms is by far the most widely used [7-12]. This approach to reclaiming contaminated land reduces the threat to groundwater and enhances the rate of biodegradation [13]. The technology commonly used for the soil remediation includes mechanical, burying, evaporation, dispersion, and washing. However, these technologies are expensive and can lead to incomplete decomposition of contaminants $[2,6,14$, 15].

Mathematical model is regarded as a decision tool that assists decision makers in effectively dealing with complex issues such as oil spillage on soil surfaces [1]. When oil is released into the subsurface, it travels downward until it 
TABLE 1: Typical soil properties.

\begin{tabular}{lccc}
\hline Soil type & Particle diameter $(\mathrm{mm})$ & Porosity, $\varepsilon_{p}$ & Permeability, $k\left(\mathrm{~m}^{2}\right)$ \\
\hline Clay & $<0.002$ & $0.4-0.7$ & $10^{-18}-10^{-15}$ \\
Silty & $0.002-0.05$ & $0.35-0.5$ & $10^{-18}-10^{-12}$ \\
Sandy & $0.05-2.00$ & $0.25-0.5$ & $10^{-14}-10^{-10}$ \\
Gravel & $<2$ & $0.25-0.4$ & $10^{-18}-10^{-10}$ \\
\hline
\end{tabular}

TABLE 2: Typical first-order biodegradation rate constants from field studies.

\begin{tabular}{|c|c|c|c|c|c|c|}
\hline \multirow{2}{*}{$\begin{array}{l}\text { Field study } \\
\text { location }\end{array}$} & \multirow{2}{*}{$\begin{array}{l}\text { Shoreline } \\
\text { type }\end{array}$} & \multirow{2}{*}{ Oil type } & \multirow{2}{*}{ Treatment } & \multicolumn{2}{|c|}{ First-order biodegradable rate day ${ }^{-1}$} & \multirow{2}{*}{ Reference } \\
\hline & & & & Alkanes & PAHs & \\
\hline \multirow{3}{*}{ Delaware } & \multirow{3}{*}{ Sandy beach } & \multirow{3}{*}{ Bonny light crude oil } & Control & 0.026 & 0.021 & \multirow{3}{*}[12]{} \\
\hline & & & Nutrient & 0.056 & 0.031 & \\
\hline & & & Inoculum & 0.045 & 0.3026 & \\
\hline \multirow{2}{*}{ Quebec, Canada } & \multirow{2}{*}{$\begin{array}{l}\text { Tidal fresh } \\
\text { water }\end{array}$} & \multirow{2}{*}{ Mesa light crude oil } & Control & 0.0028 & 0.0028 & \multirow{2}{*}{ [19] } \\
\hline & & & Nutrient & $0.0023-0.034$ & $0.0016-0.041$ & \\
\hline \multirow{5}{*}{ Texas } & \multirow{5}{*}{$\begin{array}{l}\text { Brackish } \\
\text { Wetland }\end{array}$} & \multirow{2}{*}{$\begin{array}{l}\text { Phase II: Arabian } \\
\text { light crude oil }\end{array}$} & Control & 0.019 & 0.017 & \multirow{5}{*}[20]{} \\
\hline & & & Nutrient & $0.042-0.061$ & $0.018-0.027$ & \\
\hline & & \multirow{3}{*}{$\begin{array}{l}\text { Phase III: Arabian } \\
\text { medium crude oil }\end{array}$} & Control & 0.020 & 0.015 & \\
\hline & & & Nutrient & 0.024 & 0.013 & \\
\hline & & & Inoculum & $0.019-0.030$ & $0.016-0.017$ & \\
\hline
\end{tabular}

encounters a low permeability layer that it cannot penetrate because of large capillary force. If oil spill is present in sufficient quantity the oil may pool on the low permeability materials or more latterly, following the geological gradient of this materials contact of oil with groundwater results in dissolution of the soluble content of oil and subsequent contamination of the groundwater [16].

Most mathematical models follow a mathematical structure that can be used to describe and study a real solution. Hence, mathematical model is a representation in mathematical terms of certain aspects of nonmathematic system. The arts and crafts of mathematical modelling are exhibited in the construction of models that not only are consistent in themselves and mirror the behaviours of their prototype, but serve some exterior purpose.

\section{Data Collection}

In this paper it is intended to describe the bioremediation of land on which oil has spilled using a mathematical model such that it is possible to predict the residual concentration of the oil at any time. The model was divided into parts which includes the following physical principles [17], that is,

(i) law of conservation of mass (continuity equation),

(ii) Darcy's diffusion in liquids (Fick's laws),

(iii) Monod kinetics.

In a porous fluid flow medium, the most significant quantity that must be conserved is the mass. In the case of oil biodegradation, no oil is generated; only consumption by microbes takes place. We shall adopt experimentally verified parameters shown in Tables $1-4$. Table 1 gives the soil properties of the polluted soil that shall be assumed. Table 2 illustrates the first-order biodegradation rate constants from field studies. Table 3 shows the relations for diffusion in porous solids. Table 4 reveals the maximum recorded growth rates for some organisms measured near their respective optimal temperature in complex media.

Other Useful Data. Approximate predictions of Tortuosity factors for common adsorbents are as follows [18]:

$$
\begin{aligned}
& \tau_{p}=\frac{\left(2-\varepsilon_{p}\right)^{2}}{\varepsilon_{p}} \quad \text { [Mackie and Meares], } \\
& \tau_{p}=\frac{1}{\varepsilon_{p}} \quad \text { [Wakao and Smith], } \\
& \tau_{p}=\varepsilon_{p}+1.5\left(1-\varepsilon_{p}\right) \quad \text { [Suzuki and Smith]. }
\end{aligned}
$$

This means that, for sandy soil, with irregular particle and pores shape and size, the tortuosity value will be much higher than the highest one from these correlations:

(a) Specific gravity of crude oil (sour blend): 0.842 .

(b) Dynamic viscosity: $34.8 \times 10^{-3} \mathrm{~Pa} \cdot \mathrm{s}$.

(c) Approximately apparent diffusivity of oil sample: $1.018 \times 10^{-3} \mathrm{~cm}^{2} / \mathrm{s}$.

For this work, the following parameters were used:

Soil type: sandy.

Porosity, $\varepsilon_{p}: 0.43$.

Tortuosity factor: 6.0. 
TABLE 3: Relations for diffusion in porous solids [18].

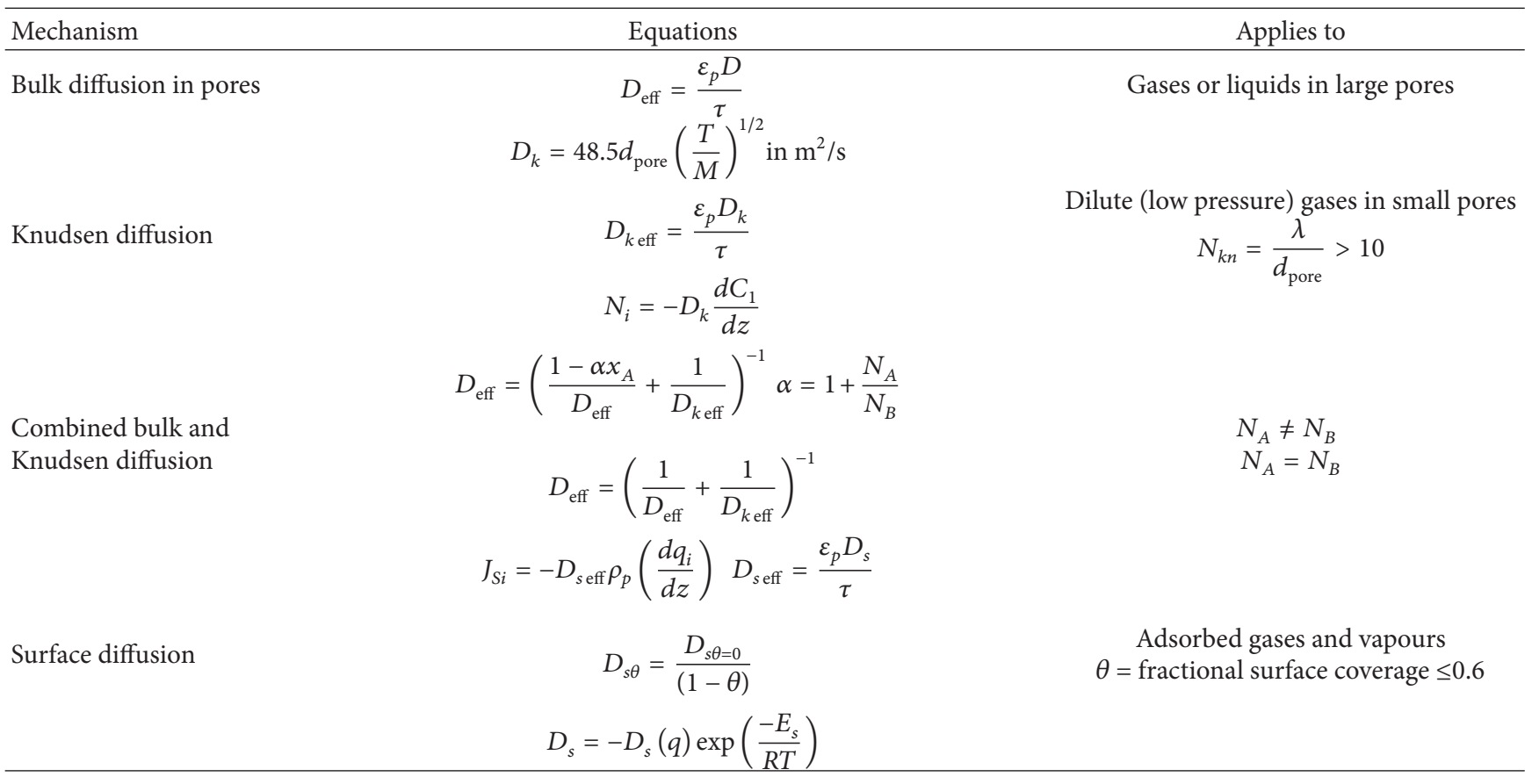

TABLE 4: Maximum recorded growth rates for some organisms measured near their respective optimal temperature in complex media [16].

\begin{tabular}{lcc}
\hline Organism & Temperature, ${ }^{\circ} \mathrm{C}$ & Doubling Time, $t_{d}(\mathrm{~h})$ \\
\hline Vibrio natriegens & 37 & 0.16 \\
Bacillus stearothermophilus & 60 & 0.43 \\
Escherichia coli & 40 & 0.38 \\
Bacillus subtilis & 40 & 0.43 \\
Pseudomonas putida & 30 & 0.75 \\
Vibrio marinus & 15 & 1.35 \\
Rhodobacter sphaeroides & 30 & 2.2 \\
\hline
\end{tabular}

Growth rate organism: $5.324 \times 10^{-7} / \mathrm{s}$ (hydrocarbon utilizers).

Soil initial concentration $[C]=0.38 \mathrm{~g} / \mathrm{g}$; soil concentration in $\mathrm{g} / \mathrm{g}$ soil can be converted to $\mathrm{g} / \mathrm{m}^{3}$ by multiplying the former by soil bulk density.

\section{Theories of Formulation}

Under this section, we considered the full formulations of Fick's and Darcy's law. Darcy's law expresses the fact that the volumetric flow rates per unit cross-sectional area at any point in a uniform porous medium are proportional to the gradient in potential in the direction of flow at that point [17]. The law is valid for laminar flow at low Reynold's number and its mathematical expression is

$$
u=\frac{k p}{\mu} \Delta \phi
$$

where $\phi=\int_{P o}^{p} d p / p+g z$ (Hubbest).
Equation (2) is generated as follows:

For a one-dimensional flow of a fluid, it may be stated as

$$
Q=A K \frac{D h}{Z} .
$$

The differential form is shown below:

$$
Q=A K \frac{d h}{d z}=-A K \frac{d[(P / P g)+Z]}{d z} .
$$

The negative sign shows that the head decreases in the direction of flow.

Dividing (4) by the flow area will give Darcy's law; thus,

$$
V=\frac{Q}{A}=-K \frac{d[(P / P g)+Z]}{d z} .
$$

For liquids other than water, the hydroid conductivity can be replaced by the permeability of the porous media, such that

$$
V=\frac{K p g}{\mu} \frac{d[(P / P g)+Z]}{d z} .
$$


This gives

$$
V=-\frac{K}{\mu}=\frac{d[P+Z p g]}{d z},
$$

where $K=K p g / \mu$ and $K$ is the permeability. $Z p g$ is a constant and its differential is zero.

Therefore (7) becomes

$$
V=-\frac{K}{\mu} \frac{d p}{d z} .
$$

Thus, for flow in the $x, y$, and $z$ directions, respectively, Darcy's law can be experienced.

$$
\begin{aligned}
& u_{x}=\frac{K x}{\mu} \frac{d p}{d x}, \\
& u_{y}=\frac{-K y}{\mu} \frac{d p}{d y}, \\
& u_{z}=\frac{-K z}{\mu}\left[\frac{d p}{d z}+\rho\right],
\end{aligned}
$$

where $u$ is volumetric rate of flows per unit cross-sectional area. $\mu$ is viscosity of the fluid. $K$ is permeability $\left(\mathrm{M}^{2}\right) . \rho$ is density of the fluid $\left(\mathrm{kg} / \mathrm{m}^{3}\right) . Z$ is height. $u_{x}$ is mass-average velocity in the $x$-direction $(\mathrm{m} / \mathrm{s}) . u_{y}$ is mass-average velocity in the $y$-direction $(\mathrm{m} / \mathrm{s}), u_{z}$ is mass-average velocity in the $z$-direction $(\mathrm{m} / \mathrm{s}) . V$ is Darcy's flux $(\mathrm{m} / \mathrm{s})$.

Fick's law deals with movement of individual molecules through a substance by virtue of their thermal energy. Diffusion occurs in substances as a result of concentration gradient; materials move from regions of higher concentration to regions of lower concentration to attain uniformity in substances. The rate at which a solute moves at any point in any direction must therefore depend on this concentration gradient at that point and in that direction. This rate is measured in a direction normal to the diffusion.

The diffusivity or diffusion coefficient, $D_{A B}$, of a constituent $A$ in solution is $B$, which is a measure of its diffusive mobility.

$$
J_{A}=-D_{A B} \frac{\partial C_{A}}{\partial_{z}}=-c D_{A B} \frac{\partial X_{A}}{\partial z} .
$$

The negative signs emphasize that diffusions occur in the direction of a drop in concentration [17].

Monod Kinetic. Monod kinetics describe

(a) Enzymes-substrate activity

(b) Adsorption isotherm behaviours

(c) Microbial growth rate

(d) Microbial uptake of substrate

The Monod equation is

$$
\mu_{k}=\frac{\mu_{\max } S}{K_{S}+S}
$$

where $\mu_{\max }$ is maximum growth rate achievable when $S \gg$ $K_{S}$. $S$ is substrate concentration. $K_{S}$ is value of the concentration of the concentration at which the specific growth rate has half its value.

Simple first-order degradation models could be written as

$$
R=\mu C,
$$

where $R$ is model reaction term. $\mu$ is first-order degradation rate coefficient. $C$ is substrate concentration.

From Figure 1, the continuity equation for flow could be written as

(Rate of oil Entering elemental Volume)

- (Rate of mass of oil leaving the element mass)

- (Consumption of oil by microbes)

$=$ (The mass rate of accumulation),

(Rate of oil entering the elemental volume)

- (Consumption of oil in the elemental volume)

$=($ Rate of accumulation of oil in the elemental volume $)$.

The net rate oil entering the elemental volume equals difference between the rate of oil entering the elements and the rate of mass of oil leaving the elemental volume.

This is given by the summation of the net mass gains in the $X, Y$, and $Z$-direction.

Let the elemental volume $=\Delta_{x} \Delta_{y} \Delta_{z}=\left[\mathrm{M}^{3}\right]$.

Let the molecular weight of oil $=M_{w o}=[\mathrm{kg} / \mathrm{mol}]$.

Let the molar flux of oil $=N_{o}\left[\mathrm{~mol} / \mathrm{m}^{2} \mathrm{~s}\right]$.

The mass rate of flow of component $A$ (oil) into the three faces with a common corner at $A$ is therefore written as

$$
\begin{aligned}
& M_{w 0}\left[\left(N_{0 x}\right)_{x} \Delta y \Delta z+\left(N_{0}, y\right) y \Delta x \Delta z\right. \\
& \left.\quad+\left(N_{0} z\right) \Delta x \Delta y\right],
\end{aligned}
$$

where $N_{0, x}$ signifies the $X$-directed flux and $\left(N_{0, x}\right)$ its value at location $X$.

Similarly, the mass rate of flow out of the three faces with a common corner at $B$ is

$$
\begin{aligned}
& M_{w 0}\left[\left(N_{0 x}\right)_{x+\Delta x} \Delta y \Delta z+\left(N_{0}, y\right)_{y+\Delta y} \Delta x \Delta z\right. \\
& \left.\quad+\left(N_{0} z\right)_{z+\Delta z} \Delta x \Delta y\right] .
\end{aligned}
$$

Since the net rate oil entering the elemental volume is the difference between the entering and leaving oil flows rates (14) and (15) become

$$
\begin{aligned}
& M_{w 0}\left[\left[\left(N_{0 x}\right)_{x+\Delta x}-\left(N_{0}, k\right)_{k}\right] \Delta y \Delta z+\left(N_{0}, y\right)_{y+\Delta y}\right. \\
& \left.-\left(N_{0} y\right) \Delta x \Delta y+\left[\left(N_{0}, z\right)_{z+\Delta z}-\left(N_{0}, z\right)_{z}\right] \Delta x \Delta y\right] .
\end{aligned}
$$

If, in addition, the rate of biochemical reaction of oil in the element volume is given as

$$
R_{0}=\frac{\mathrm{Mol}}{\text { (volume) (time) }}=\frac{\mathrm{Mol}}{\mathrm{M}^{2} S},
$$




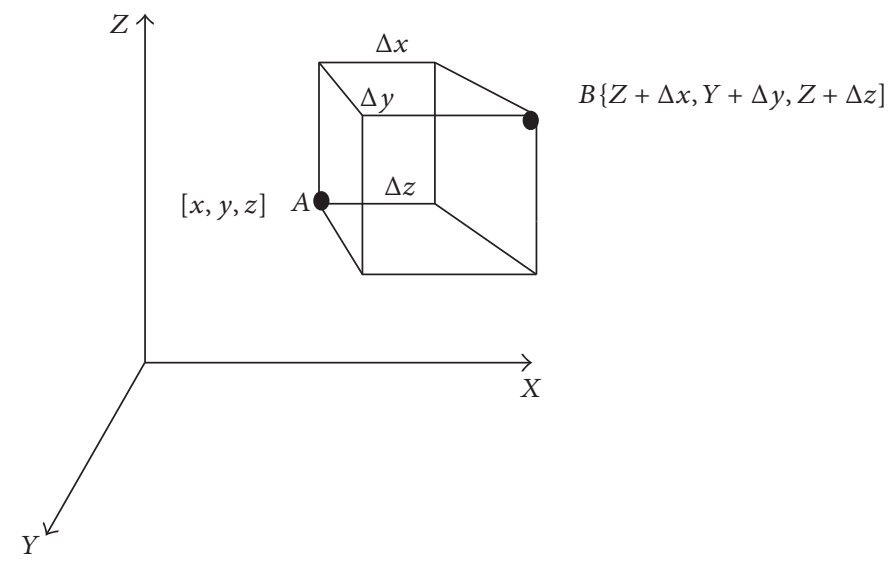

FIGURE 1: Elemental volume of oil contaminated soil.

therefore its production rate is

$$
M_{w o} R_{0} \Delta x \Delta y \Delta_{2}\left[\frac{\text { mass }}{\text { time }}\right] .
$$

The rate of accumulation with the soil elemental volume is given by the following.

If the total oil in the elemental volume $=P_{o}=\Delta_{x} \Delta_{y} \Delta_{z} P_{o}$, therefore its rate of accumulation is

$$
\Delta_{x} \Delta_{y} \Delta_{z} \frac{d p o}{d t}
$$

Combining (16), (18), and (19), dividing by $\Delta_{x} \Delta_{y} \Delta_{z}$, and taking the limit as the three distances become zero give

$$
\begin{aligned}
& M_{w 0} {\left[\frac{\partial N_{0}, x}{\partial x} \Delta x \Delta y \Delta z+\frac{\partial N_{0}, y}{\partial y} \Delta x \Delta y \Delta z\right.} \\
&+\left.\frac{\partial N_{0} z}{\partial_{2}} \Delta x \Delta y \Delta z\right]-M_{w o} R_{0} \Delta x \Delta y \Delta z \frac{\partial p o}{\partial t} \\
&= \Delta x \Delta y \Delta z \frac{\partial p o}{\partial t}, \\
& M_{w 0} {\left[\frac{\partial N_{0}, x}{\partial x}+\frac{\partial N_{0} y}{\partial y}+\frac{\partial N_{0}, z}{\partial z}\right] \Delta x \Delta y \Delta z-M_{w o} } \\
&=\frac{R o \partial p o}{\partial t} \Delta x \Delta y \Delta z=\frac{\partial p o}{\partial t} \Delta x \Delta y \Delta z, \\
& M_{w 0} {\left[\frac{\partial N_{0}, x}{\partial x}+\frac{\partial N_{0} y}{\partial y}+\frac{\partial N_{0}, z}{\partial z}\right] M_{w o} \frac{R o \partial p o}{\partial t} } \\
&= \frac{-\partial p o}{\partial t} \\
& \therefore M_{w 0}\left[\frac{\partial N_{0}, x}{\partial x}+\frac{\partial N_{0} y}{\partial y}+\frac{\partial N_{0}, z}{\partial z}\right]+\frac{\partial p o}{\partial t} \\
&=-M_{w o} R_{0} .
\end{aligned}
$$

The molar flux of the oil $N_{0}$ is made up of two parts: The adjusted amount or rate from the bulk motion $B_{o}$ and the fraction $X_{A}$ of $N$ which is $B_{o}$ and resulting from diffusion $J_{A}$

$$
N_{o}=B_{o}+J_{o} \text {. }
$$

Equation (22) expressed in terms of mass in the $X$-direction gives

$$
M_{w 0} N_{o} x=U_{x} P o+M_{w 0} J_{o x}
$$

where $U_{x}$ is the mass-average velocity

$J_{0}$ is the diffusion flux given by Fick's law

$$
J_{0} x=-D o \frac{\partial}{\partial x}
$$

Differentiating (23), we have

$$
M_{w 0} \frac{\partial N_{o} y}{\partial x}=U x \frac{\partial p o}{\partial x}+P o \frac{\partial U x}{\partial x}+M_{w 0} \frac{\partial J_{0}, x}{\partial x}
$$

Similarly written for $y$ and $z$-direction, we have

$$
\begin{aligned}
& M_{w 0} \frac{\partial N_{o} y}{\partial y}=U y \frac{\partial p o}{\partial y}+P o \frac{\partial U y}{\partial y}+M_{w 0} \frac{\partial J_{0}, y}{\partial y} \\
& M_{w 0} \frac{\partial N_{0} z}{\partial z}=U z \frac{\partial P o}{\partial z}+P o \frac{\partial U_{z}}{\partial z}+M_{w o} \frac{\partial J_{o} z}{\partial z}
\end{aligned}
$$

Substituting (25) and (26)

$$
\begin{gathered}
P o\left[\frac{\partial u x}{\partial x}+\frac{\partial u y}{\partial y}+\frac{\partial u_{z}}{\partial z}\right] \\
+\underbrace{U x \frac{\partial p o}{\partial x}+U y \frac{\partial p o}{\partial y}+U z \frac{\partial p o}{\partial z}}_{a} \\
+M_{w 0} \underbrace{\left[\frac{\partial J_{o} x}{\partial x}+\frac{\partial J_{o}, y}{\partial y}+\frac{\partial J_{o}, z}{\partial z}\right]}_{b}+\underbrace{\frac{\partial p o}{\partial t}}_{c} \\
=-M_{w o} \underline{R_{o} .}
\end{gathered}
$$


Let us assume that density and flux are constants; then $a, b, c$ $=0$, so we have

$$
\begin{aligned}
P o\left[\frac{\partial u x}{\partial x}+\frac{\partial u y}{\partial y}+\frac{\partial u_{z}}{\partial z}\right] & =-M_{w o} R o, \\
\frac{p_{0}}{M_{w 0}} & =C .
\end{aligned}
$$

Substituting for $J_{0} x, J_{0} y \& J_{0} z$ in (24) and assuming that the diffusivity $D_{o}$ is equal to the $x$-, $y$-, and $z$-direction therefore (27) gives

$$
\begin{aligned}
& {\left[U x \frac{\partial p o}{\partial x}+U y \frac{\partial p o}{\partial y}+U z \frac{\partial p o}{\partial z}\right]} \\
& \quad+P o\left[\frac{\partial u k}{\partial x}+\frac{\partial u y}{\partial y}+\frac{\partial u_{z}}{\partial z}\right] \\
& \quad-M_{w o} D o\left[\frac{\partial^{2} C}{\partial x^{2}}+\frac{\partial^{2} C}{\partial y^{2}}+\frac{\partial^{2} C}{\partial z^{2}}\right]+\frac{\partial p o}{\partial t} \\
& =-M_{w o} R_{o} .
\end{aligned}
$$

Dividing by molecular weight, we have

$$
\begin{gathered}
{\left[U x \frac{\partial c}{\partial x}+U y \frac{\partial c}{\partial y}+U z \frac{\partial x}{\partial z}\right]+C\left[\frac{\partial u x}{\partial x}+\frac{\partial u y}{\partial y}+\frac{\partial u_{z}}{\partial z}\right]} \\
-D o\left[\frac{\partial^{2} C}{\partial x^{2}}+\frac{\partial^{2} C}{\partial y 2}+\frac{\partial^{2} C}{\partial z^{2}}\right]+\frac{\partial C}{\partial t}=-R_{o} \\
\frac{\partial U x}{\partial x}+\frac{\partial U y}{\partial y}+\frac{\partial U z}{\partial z}=0 \quad \text { [Continuity equation] } \\
\therefore\left[U x \frac{\partial c}{\partial x}+U y \frac{\partial c}{\partial y}+U z \frac{\partial c}{\partial z}\right] \\
-D o\left[\frac{\partial^{2} C}{\partial x^{2}}+\frac{\partial^{2} C}{\partial y^{2}}+\frac{\partial^{2} C}{\partial z^{2}}\right]+\frac{\partial C}{\partial t}=-R_{o} .
\end{gathered}
$$

Assuming that velocity $=0$, there is no chemical reaction

Equation (31) reduces to Fick's law:

$$
\frac{\partial c}{\partial t}=D o\left[\frac{\partial^{2} C}{\partial x^{2}}+\frac{\partial^{2} C}{\partial y^{2}}+\frac{\partial^{2} C}{\partial z^{2}}\right] .
$$

Assuming a unidirectional diffusion in a particular dimension is taken as $z$-direction, (31) becomes

$$
\begin{gathered}
\frac{U z \partial c}{\partial z}-D o \frac{\partial^{2} C}{\partial z^{2}}+\frac{\partial C}{\partial t}=-R_{o} \\
\frac{\partial c}{\partial z}-D o \frac{\partial^{2} C}{\partial z^{2}}+\frac{U z \partial C}{\partial t}-R_{o}
\end{gathered}
$$

where $R_{o}$ is the rate of consumption of oil by microorganism which can be equivalent to " $\mu$." $\mu$ is the growth rate of the microorganism.
Monod kinetics describes the growth rate of organism; that is,

$$
\mu=\frac{\mu_{\max } C}{K_{o}+C},
$$

where $K_{o}$ is saturation constant.

The obtained mathematical model is

$$
\frac{\partial c}{\partial t}=D=\frac{\partial^{2} C}{\partial Z^{2}}-U z \frac{\partial C}{\partial z}-\mu C .
$$

\section{Model Decomposition}

The methods used for this study are listed below:

(i) Conversion of variables to dimensionless form.

(ii) Discretion of model.

4.1. Dimensionless Parameter Determination. The obtained mathematical model is

$$
\frac{\partial C}{\partial t}=D \frac{\partial^{2} C}{\partial z^{2}}-u_{z} \frac{\partial C}{\partial z}-\mu C
$$

The expression above is an initial value problem in terms of time, $t$, but boundary conditions can be generated for the variable $z$.

Initial conditions are

$$
\begin{aligned}
C & =C_{0}, \\
z & =0, \\
t & =0 ; \\
C & =0, \\
z & >0, \\
t & =0 .
\end{aligned}
$$

Boundary conditions are

$$
\left.D \frac{\partial C}{\partial t}\right|_{z=0, t>0}=\left(u_{z} C_{0}-u_{z} C\right)+\left.D \frac{\partial^{2} C}{\partial z^{2}}\right|_{z=L, t>0}=0,
$$

where $C_{0}$ is initial oil concentration and $L$ is total height.

Defining the dimensionless variables,

$$
\begin{aligned}
& a a=\frac{D}{L^{2}}, \\
& a b=\frac{-u_{z}}{L}, \\
& a c=-\mu,
\end{aligned}
$$

so that the model equation (21) becomes

$$
\frac{d \bar{c}}{d t}=a a \frac{\partial^{2} \bar{c}}{\partial z^{2}}+a b \frac{\partial \bar{c}}{\partial z}+a c \bar{c},
$$

where $\bar{c}=C / C_{0}$ is relative (dimensionless) concentration. 
Considering the nature of the boundary condition, $D(\partial C / \partial z)=0$, at $z=0$, the transformation below is used $z=z^{-2}$

$$
d z=2 z d z
$$

So that:

$$
\begin{aligned}
\frac{\partial \bar{c}}{\partial z} & =\frac{\partial \bar{c}}{\partial z} * \frac{\partial z}{\partial z} \\
& =2 z \frac{\partial \bar{c}}{\partial z} \\
& =2 \sqrt{ } z \frac{\partial \bar{c}}{\partial z}
\end{aligned}
$$

Also,

$$
\frac{\partial^{2} \bar{c}}{\partial z^{2}}=\frac{\partial}{\partial z}\left[\frac{\partial \bar{c}}{\partial z}\right]
$$

This, from (39c), becomes

$$
\begin{aligned}
\frac{\partial}{\partial z}\left[2 \sqrt{ } z \frac{\partial \bar{c}}{\partial z}\right] & =2\left[\frac{1}{2 \sqrt{ } z} \frac{\partial \bar{c}}{\partial z}+\sqrt{ } z \frac{\partial^{2} \bar{c}}{\partial z^{2}}\right] \\
& =2 \sqrt{ } z \frac{\partial^{2} \bar{c}}{\partial z^{2}}+\frac{1}{\sqrt{ } z} \frac{\partial \bar{c}}{\partial z} .
\end{aligned}
$$

Substituting (39c) and (40b) into (37) yields

$$
\frac{d \bar{c}}{d t}=2 a a \sqrt{ } z \frac{\partial^{2} \bar{c}}{\partial z^{2}}+\frac{a a}{\sqrt{ } z} \frac{\partial \bar{c}}{\partial z}+2 a b \sqrt{ } z \frac{\partial \bar{c}}{\partial z}+a c \bar{c}
$$

This can be factorized to give

$$
\frac{d \bar{c}}{d t}=2 a a \sqrt{ } z \frac{\partial^{2} \bar{c}}{\partial z^{2}}+\sqrt{ } z\left[\frac{a a}{z}+2 a b\right] \frac{\partial \bar{c}}{\partial z}+a c \bar{c} .
$$

The coefficients in this expression can be by single terms such that

$$
\begin{aligned}
& a_{1}=2 a a \sqrt{z} \\
& a_{2}=\sqrt{z\left[\frac{a a}{z}+2 a b\right]}, \\
& a_{3}=a c,
\end{aligned}
$$

to give new ODE

$$
\frac{d \bar{C}}{d t}=a_{1} \frac{\partial^{2} \bar{C}}{\partial z^{2}}+a_{2} \frac{\partial \bar{C}}{\partial z}+a_{3} \bar{C}
$$

Rendering the initial conditions (at $t=0$ ) dimensionless yields

$$
\begin{aligned}
\bar{C} & =1, \\
\bar{z} & =0, \\
t & =0 ; \\
\bar{C} & =0, \\
\bar{z} & >0, \\
t & =0 .
\end{aligned}
$$

Rendering the other boundary conditions (at $z=L$ ) dimensionless yields

$$
a d \frac{\partial \bar{C}}{\partial \bar{z}}=\bar{C}-1
$$

where $a d=-a a / a b$.

4.2. Discretization of Model. Orthogonal collocation is applied to boundary value problem. In this case, " $z$ " is bounded but " $t$ " is not. Carrying out discretization, the following transformations are used:

$$
\begin{gathered}
\frac{\partial \bar{C}}{\partial z}=\sum_{1}^{N+1} A_{i \cdot j} \bar{C}_{j}, \\
\frac{\partial^{2} \bar{C}}{\partial z^{2}}=\sum_{1}^{N+1} B_{i . j} \bar{C}_{j} .
\end{gathered}
$$

Substituting these expressions into (43) yields

$$
\frac{d \bar{C}_{i}}{d t}=a_{i} \sum_{1}^{N+1} B_{i j} \bar{C}_{j}+a_{2} \sum_{1}^{N+1} A_{i j} \bar{C}_{j}+a_{3} \bar{C}_{i} .
$$

This can be written as

$$
\begin{aligned}
\frac{d \bar{C}_{i}}{d t}= & +a_{1} \sum_{1}^{N} B_{i j} \bar{C}_{j}+a_{1} B_{i, N+1} \bar{C}_{N+1}+a_{2} \sum_{1}^{N} A_{i j} \bar{C}_{j} \\
& +a_{2} A_{i, N+1} \bar{C}_{N+1}+a_{3} \bar{C}_{i} .
\end{aligned}
$$

And by grouping like terms gives

$$
\begin{aligned}
& \frac{d \bar{C}_{i}}{d t} \sum_{1}^{N}\left(a_{1} B_{i j}+a_{2} A_{i j}\right) \bar{C}_{j} \\
& \quad+\left(a_{1} B_{i, N+1}+a_{2} A_{i, N+1}\right) \bar{C}_{N+1}+a_{3} \bar{C}_{i} .
\end{aligned}
$$

Discretizing the other boundary condition (at $z=L$ ) yields

$$
a_{4 \sum_{1}^{N+1} A_{N+1, j}} \bar{C}_{j}=\bar{C}_{N+1}-1,
$$

where $a_{4}=2 a b \sqrt{z}$ (from (39c) and (44a)).

As in (46a), (47a) can be expanded as

$$
a_{4 \sum_{1}^{N} A_{N+1, j}} \bar{C}_{j}+a_{4} A_{N+1, N+1} \bar{C}_{N+1}=\bar{C}_{N+1}-1,
$$

so that

$$
\bar{c}_{N+1}=\frac{a_{4} \sum_{1}^{N} A_{N+1, j} \bar{C}_{j}+1}{\left(1-a_{4} A_{N+1, N+1}\right)}
$$


which when substituted into (46b) gives the expression

$$
\begin{aligned}
\frac{d \bar{C}_{i}}{d t} & \\
= & \sum_{1}^{N}\left(a_{i} B_{i j}+a_{2} A_{i J}\right) \bar{C}_{j} \\
& +\frac{\left(a_{i} B_{i, N+1}+a_{2} A_{i, N+1}\right)\left(a_{4} \sum_{1}^{N} A_{N+1, j} \bar{C}_{j}+1\right)}{1-a_{4} A_{N+1, N+1}} \\
& +a_{3} \bar{c}_{i} .
\end{aligned}
$$

In simple terms, the expression above can be written as

$$
\frac{d \bar{C}_{i}}{d t}=\sum_{1}^{N}\left(f_{i j} \bar{C}_{j}+e_{i}+a_{3} \bar{C}_{i}\right),
$$

where

$$
\begin{aligned}
f_{i j} & =a_{i} B_{i j}+a_{2} A_{i j} \frac{a_{4}\left(a_{1} B_{i, N+1}+a_{2} A_{i, N+1}\right)\left(A_{N+1, j}\right)}{1-a_{4} A_{N+1, N+1}}, \\
e_{i} & =\frac{a_{1} B_{i, N+1}+a_{2} A_{i, N+1}}{1-a_{4} A_{N+1, N+1}} .
\end{aligned}
$$

As a matrix, (48) could be shown as

$$
\begin{aligned}
{\left[\begin{array}{c}
C_{1} \\
C_{2} \\
M \\
C_{N}
\end{array}\right]=} & {\left[\begin{array}{llll}
f_{11} & f_{12} & K & f_{1 N} \\
f_{21} & \wedge & \wedge & f_{2 N} \\
M & \wedge & \wedge & M \\
f_{N 1} & \wedge & \wedge & f_{N N}
\end{array}\right]\left[\begin{array}{l}
C_{1} \\
C_{2} \\
M \\
C_{N}
\end{array}\right]+\left[\begin{array}{c}
e_{1} \\
M \\
M \\
e_{N}
\end{array}\right] } \\
& +a_{3}\left[\begin{array}{c}
C_{1} \\
C_{2} \\
M \\
C_{N}
\end{array}\right] .
\end{aligned}
$$

Rewriting this in vector form yields

$$
\begin{gathered}
\frac{d C}{d t}=\left(f+1 a_{3}\right) C+e, \\
{\left[\frac{d C}{d t}=f^{*} C+e\right] .}
\end{gathered}
$$

The resultant equation above representing a system of $\mathrm{N}$ coupled ordinary differential equations with initial conditions stated in (34) can be solved using a numerical integration method such as the fourth-order Runge-Kutta method.

\section{Results and Discussion}

Using (33) we obtained three different type solutions as shown in Figures 2(a)-2(f). The solution represents the various possibilities in the bioremediation process. Figure 2(a) shows how the residual oil concentration is expected to reduce with time. This solution is trivial because it expresses the normal scenario at the completion of a successful field work. Figure 2(b) shows what happens when the mass-average velocity in the $z$-direction is increased from $0.25 \mathrm{~m} / \mathrm{s}$ to $0.45 \mathrm{~m} / \mathrm{s}$. At shorter time, the residual oil concentration is expected to reduce. However, at different scenario, for example, considering a heterogeneous soil sample, the concentration may increase with time at a defined soil depth (Figures 2(c) and 2(d)). However, at varying or selective batch treatment (Figures 2(e) and 2(f)), there could be a more effective treatment. Hence we applied the model to practical or experimental results.

The residual substrate concentration reduces with time at any depth in the soil clearly indicating the processes considered in the model development (bulk flow, molecular diffusion, and microbial degradation). While experimental values show reduction from $0.38 \mathrm{~g} / \mathrm{g}$ soils to $0.14 / \mathrm{g}$ soils after 36 days, simulated values drop from $0.38 \mathrm{~g} / \mathrm{g}$ soil to $0.08 \mathrm{~g} / \mathrm{g}$ soil. These values yield comparable overall disappearance rates of $0.006 \mathrm{~g} / \mathrm{g}$ soil per day and $0.0083 \mathrm{~g} / \mathrm{g}$ soil per day, respectively (Figures 2-3).

Also, noticeable from Figure 4, the concentration of residual oil at soil depths closer to the surface rises rapidly initially before displaying the trend of concentration drop at the surface. This is as a result of more rapid accumulation in these layers than in layers farther from the surface. This trend reflects the effect of the soil properties and distance on the oil flow as aptly described by the model's boundary conditions. Reasonably, Figure 5 indicates a very small amount of oil in deeper layers of soil at the earlier time that gradually builds up over time with increasing bulk flow.

For the soil type considered in the experiment used as comparison (sandy soil), the model displays similar trend in the range of oil velocity values permissible for the soil type (varying only soil permeability). This further confirms the model developed and the computer program package for its solution, accurate tools for its solution, and accurate tools for predicting the fate of crude spilled on soil. Figure 6 shows that the concentration of residual oil at the soil depths closer to the surface rises rapidly initially before displaying the trend of concentration drop at the surface. This is as a result of more rapid accumulation in these layers than layers farther from the surface.

This trend reflects the effect of the soil properties and distance on the oil flow as described by the model's boundary conditions.

\section{Conclusion}

Bioremediation provides the best environment-friendly approach to treating oil pollution by spillage. Requirements for success in carrying out this technique of remediation include adequate knowledge of the soil characteristics nature of microorganisms that degrade crude oil (whether present in the soil or not) and ample time. Because soil types and microbial growth varies with pollutant concentration and time, bioremediation of polluted site is usually specific to the site. 


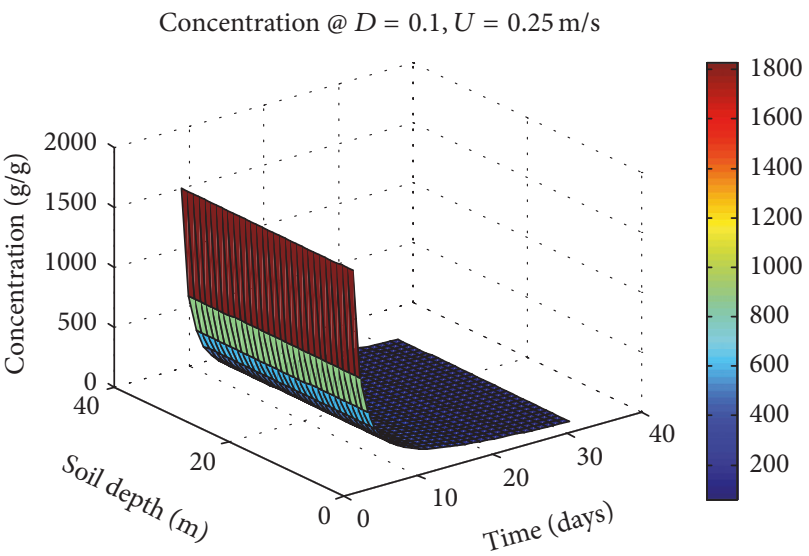

(a)

Concentration @ $D=0.1, U=0.25 \mathrm{~m} / \mathrm{s}$

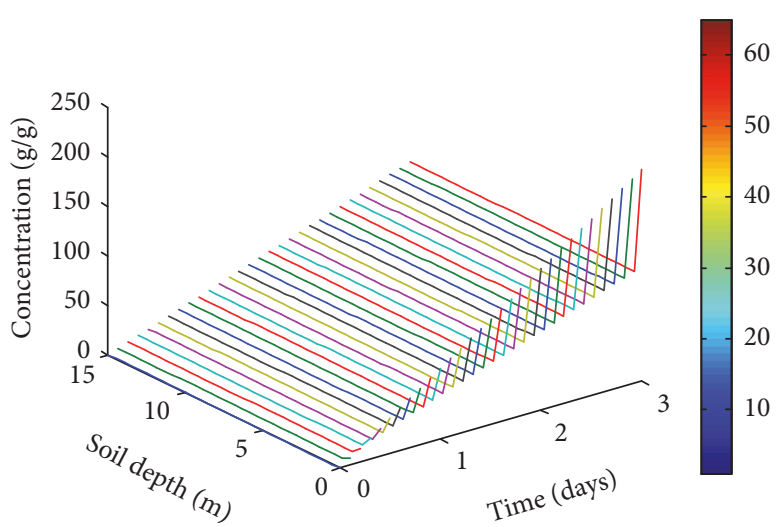

(c)

Concentration@ $D=0.1, U=0.25 \mathrm{~m} / \mathrm{s}$

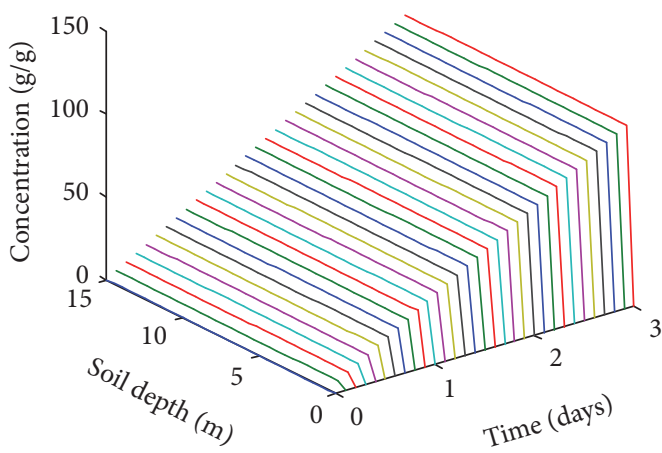

(e)

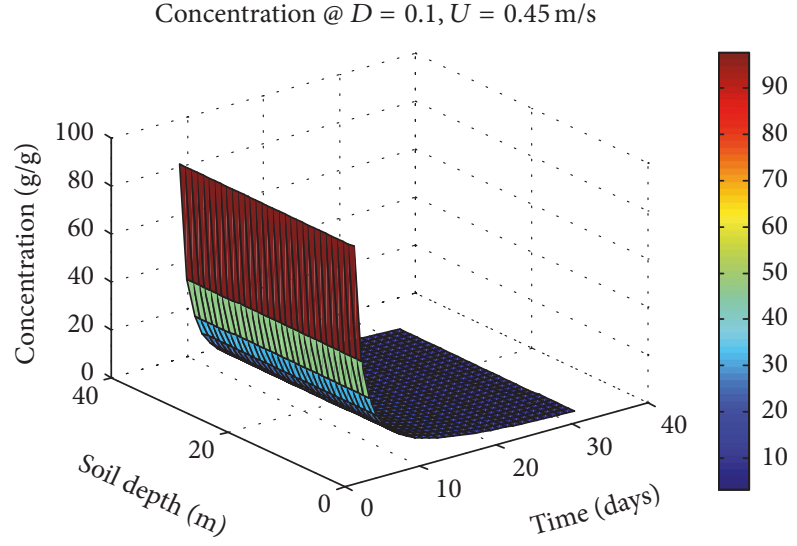

(b)

Concentration@ $D=0.1, U=0.45 \mathrm{~m} / \mathrm{s}$

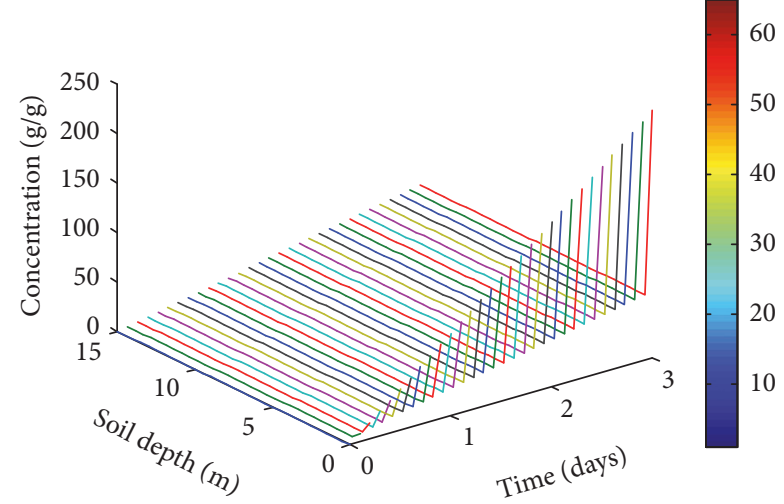

(d)

Concentration@ $D=0.1, U=0.45 \mathrm{~m} / \mathrm{s}$

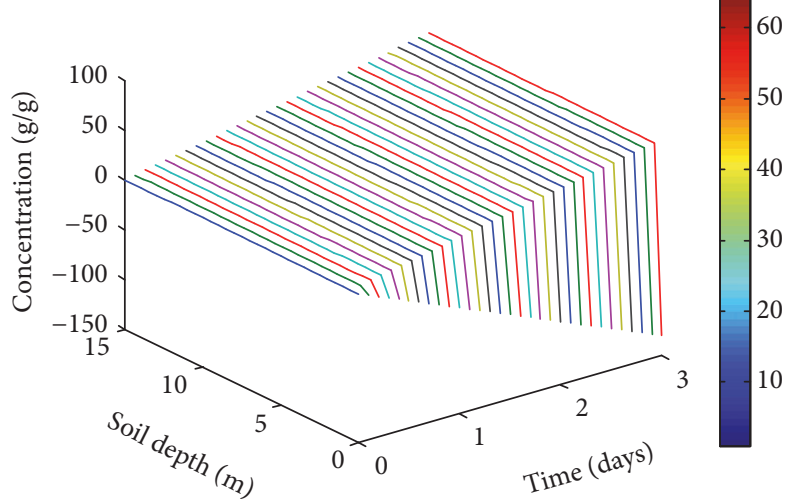

(f)

FIGURE 2: Possibilities of the bioremediation process: (a) Solution 1 at $D=0.1, U=0.25$; (b) Solution 1 at $D=0.1, U=0.45$; (c) Solution 2 at $D=0.1, U=0.25$; (d) Solution 2 at $D=0.1, U=0.45$; (e) Solution 3 at $D=0.1, U=0.25$; (f) Solution 3 at $D=0.1, U=0.45$.

With adequate data on the soil and required microbe characteristics, the right mathematical description of the process can be developed, as achieved in this work. This can be used to predict the fate of the oil with time and the extent of pollution; to predict time when complete remediation would have taken place; and to investigate possible methods of increasing the rate of bioremediation. Ultimately, the cost of bioremediation is reduced when the above roles of model development and solution are carried out.

The model developed in the course of this work suits only a simple case where no temperature effects come to play and underground water is not affected by the oil spill. The availability of all relevant experimental data plays a crucial part in comparing trend by simulation with that obtained from 


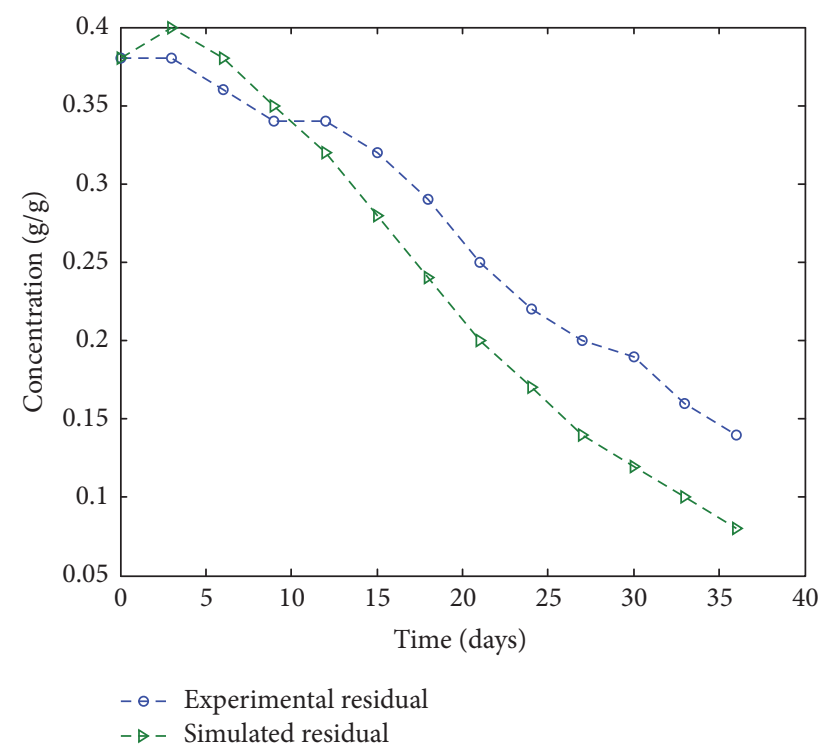

FIGURE 3: Comparative analysis of experimental and simulated results.

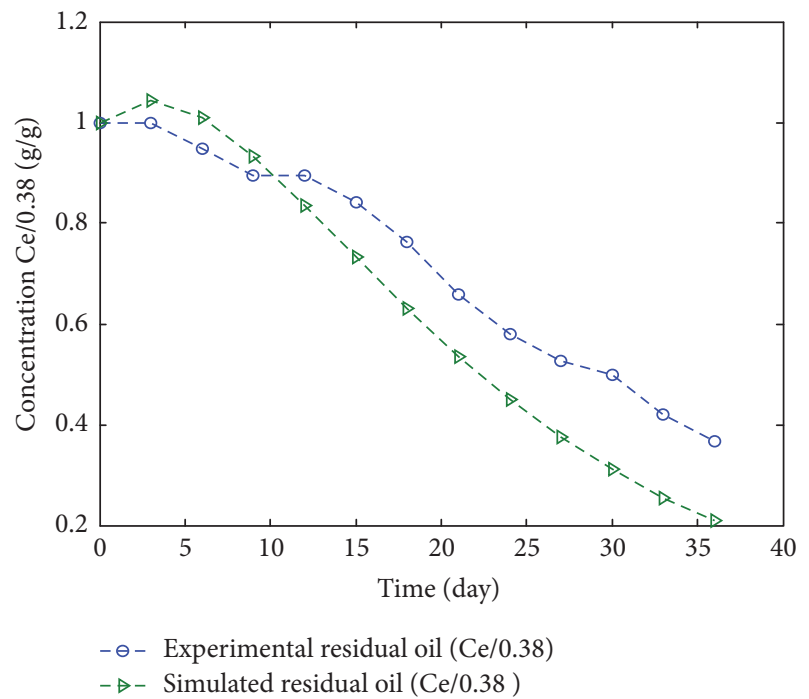

FIGURE 4: Comparative analysis of experimental and simulated results at $\mathrm{Ce} / 0.38$ (dimensionless).

experiment. In the case of bioremediation, the characteristics of the particular soil type being remediated, microbial growth rates at prevailing environmental conditions, and relevant properties of the particular oil type spilled are necessary to prove the reliability and robustness of the model and its solution method.

\section{Variables}

$k$ : Permeability $\left(\mathrm{m}^{2}\right)$

$D$ : Diffusion coefficient (length ${ }^{2} /$ time or $^{2}$ $\mathrm{cm}^{2} / \mathrm{sec}$.)

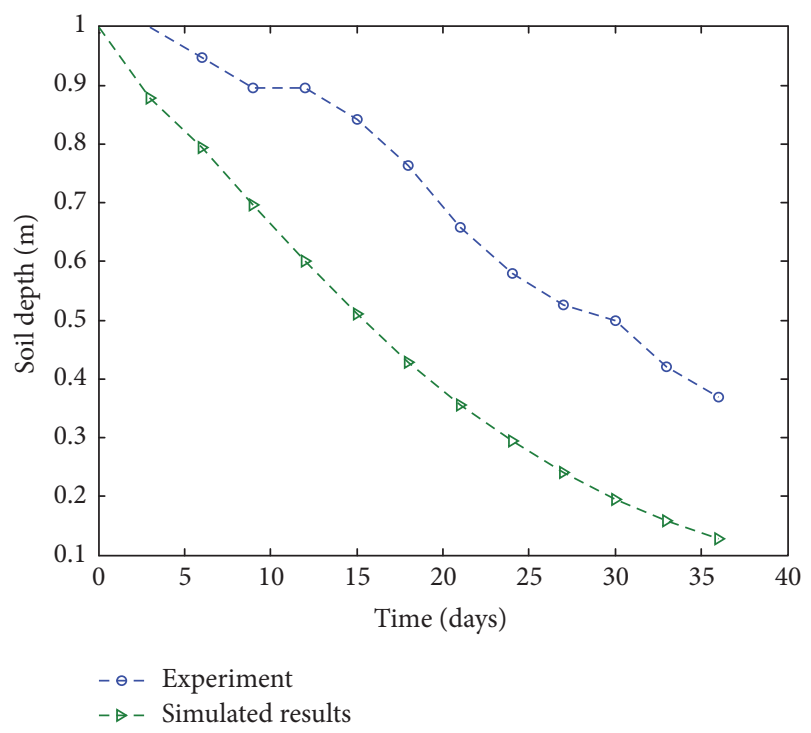

FIGURE 5: Comparative analysis of experimental and simulated results on soil depth.

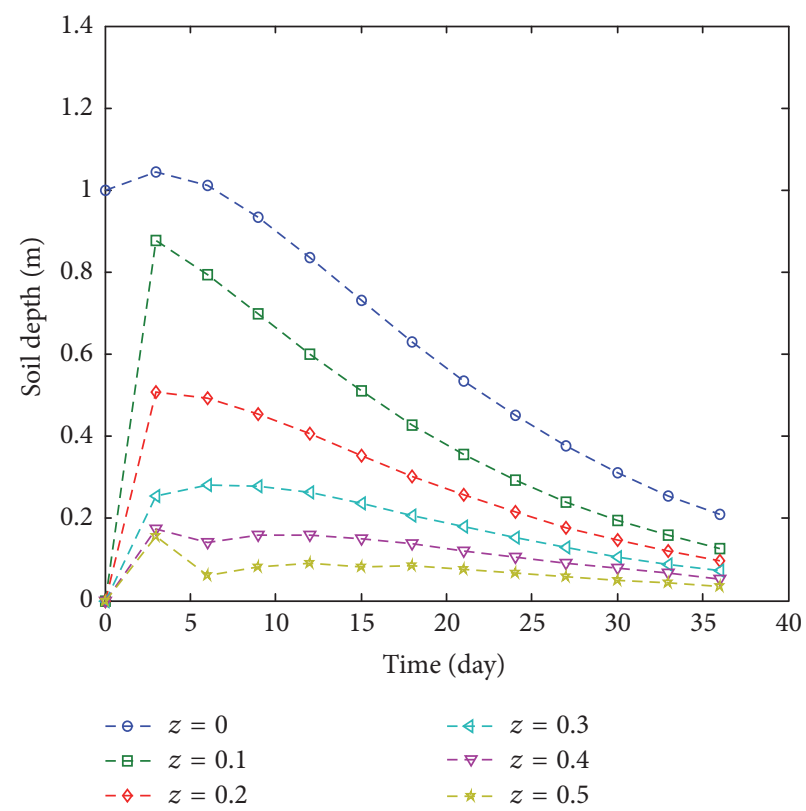

FIGURE 6: Simulated results of varying soil depths.

$D_{k}$ : Knudsen diffusion (in $\mathrm{m}^{2} / \mathrm{s}$ )

$\rho:$ Density of the fluid $\left(\mathrm{kg} / \mathrm{m}^{3}\right)$

$u$ : Velocity of the fluid $(\mathrm{m} / \mathrm{s})$

$L: \quad$ Characteristic linear dimension $(\mathrm{m})$

$\mu$ : Dynamic viscosity of the fluid with respect to object (Pa.s or $\mathrm{N} \cdot \mathrm{s} / \mathrm{m}^{2}$ or $\left.\mathrm{kg} / \mathrm{m} \cdot \mathrm{s}\right)$

Q: Volumetric flow rate $\left(\mathrm{m}^{3} / \mathrm{s}\right)$

A: Pipe's cross-sectional area $\left(\mathrm{m}^{2}\right)$

$t_{d}$ : Doubling Time (h)

$u_{x}$ : Mass-average velocity in the $x$-direction $(\mathrm{m} / \mathrm{s})$ 
$u_{y}: \quad$ Mass-average velocity in the $y$-direction $(\mathrm{m} / \mathrm{s})$

$u_{z}: \quad$ Mass-average velocity in the $z$-direction $(\mathrm{m} / \mathrm{s})$

$V: \quad$ Darcy's flux $(\mathrm{m} / \mathrm{s})$

$\mu_{\max }:$ Maximum growth rate $\left(\right.$ time $\left.^{-1}\right)$.

\section{Conflicts of Interest}

The authors declare that there are no conflicts of interest regarding the publication of this paper.

\section{Acknowledgments}

The authors appreciate the partial sponsorship of Covenant University, Ota, Nigeria.

\section{References}

[1] M. E. Amigun, Mathematical modelling of in-situ bioremediation of crude petroleum oil polluted soil [M.S. thesis], Department of Chemical Engineering, University of Lagos, Lagos, Nigeria, 2007.

[2] N. Das and P. Chandran, "Microbial degradation of petroleum hydrocarbon contaminants: an overview," Biotechnology Research International, vol. 2011, Article ID 941810, 13 pages, 2011.

[3] P.-W. Grace Liu, T. C. Chang, L.-M. Whang, C.-H. Kao, P.-T. Pan, and S.-S. Cheng, "Bioremediation of petroleum hydrocarbon contaminated soil: effects of strategies and microbial community shift," International Biodeterioration and Biodegradation, vol. 65, no. 8, pp. 1119-1127, 2011.

[4] M. D. Che, "Conservation cost estimate including investigation and monitoring expenses," in Proceedings of the Soil and Groundwater Remediation Conference, Taipei, Taiwan, 2002.

[5] T. L. Propst, R. L. Lochmiller, C. W. Qualls Jr., and K. McBee, "In situ (mesocosm) assessment of immunotoxicity risks to small mammals inhabiting petrochemical waste sites," Chemosphere, vol. 38, no. 5, pp. 1049-1067, 1999.

[6] C. Holliger, S. Gaspard, G. Glod et al., "Contaminated environments in the subsurface and bioremediation: organic contaminants," FEMS Microbiology Reviews, vol. 20, no. 3-4, pp. 517-523, 1997.

[7] C. Barbeau, L. Deschênes, D. Karamanev, Y. Comeau, and R. Samson, "Bioremediation of pentachlorophenol-contaminated soil by bioaugmentation using activated soil," Applied Microbiology and Biotechnology, vol. 48, no. 6, pp. 745-752, 1997.

[8] J. T. Dibble and R. Bartha, "Effect of environmental parameters on the biodegradation of oil sludge," Applied and Environmental Microbiology, vol. 37, no. 4, pp. 729-739, 1979.

[9] M. Eriksson, G. Dalhammar, and A.-K. Borg-Karlson, "Aerobic degradation of a hydrocarbon mixture in natural uncontaminated potting soil by indigenous microorganisms at $20^{\circ} \mathrm{C}$ and $6^{\circ} \mathrm{C}$, Applied Microbiology and Biotechnology, vol. 51, no. 4, pp. 532-535, 1999.

[10] R. E. Hinchee, J. Fredrickson, B. C. Alleman, J. V. Forsyth, Y. M. Tsao, and R. D. Bleam, "Bioremediation: when is bioaugmentation needed?" in Bioaugmentation for Site Remediation, pp. 1-14, Battelle Press, Columbus, Ohio, USA, 1995.
[11] S. J. Macnaughton, J. R. Stephen, A. D. Venosa, G. A. Davis, Y.-J. Chang, and D. C. White, "Microbial population changes during bioremediation of an experimental oil spill," Applied and Environmental Microbiology, vol. 65, no. 8, pp. 3566-3574, 1999.

[12] A. D. Venosa, M. T. Suidan, B. A. Wrenn et al., "Bioremediation of an experimental oil spill on the shoreline of Delaware Bay," Environmental Science and Technology, vol. 30, no. 5, pp. 1764$1775,1996$.

[13] S. Mishra, J. Jyot, R. C. Kuhad, and B. Lal, "Evaluation of inoculum addition to stimulate in situ bioremediation of oily-sludgecontaminated soil," Applied and Environmental Microbiology, vol. 67, no. 4, pp. 1675-1681, 2001.

[14] K. A. Kvenvolden and C. K. Cooper, "Natural seepage of crude oil into the marine environment," Geo-Marine Letters, vol. 23, no. 3-4, pp. 140-146, 2003.

[15] P. J. J. Alvarez and T. M. Vogel, "Substrate interactions of benzene, toluene, and para-xylene during microbial degradation by pure cultures and mixed culture aquifer slurries," Applied and Environmental Microbiology, vol. 57, no. 10, pp. 2981-2985, 1991.

[16] M. E. Imomoh, Mathematical modelling of in-situ bioremediation of crude petroleum pollutes soil [B.Sc. thesis], Department of Chemical Engineering UNILAG, 2006.

[17] E. T. Robert, Mass-Transfer Operations, 3rd edition, 1981.

[18] D. W. Green and R. H. Perry, Perry's Chemical Engineer's Handbook, McGraw-Hill, New York, NY, USA, 8th edition, 2007.

[19] A. D. Venosa, K. Lee, M. T. Suidan et al., "Bioremediation and biorestoration of a crude oil-contaminated freshwater wetland on the St. Lawrence river," Bioremediation Journal, vol. 6, no. 3, pp. 261-281, 2002.

[20] M. A. Simon, J. S. Bonner, T. J. McDonald, and R. L. Autenrieth, "Bioaugmentation for the enhanced bioremediation of petroleum in a wetland," Polycyclic Aromatic Compounds, vol. 14, no. 14, pp. 231-239, 1999. 


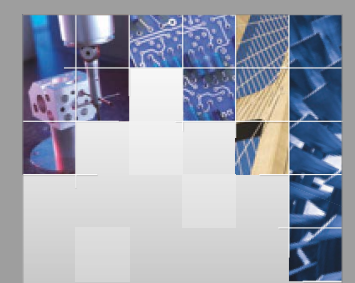

\section{Enfincering}
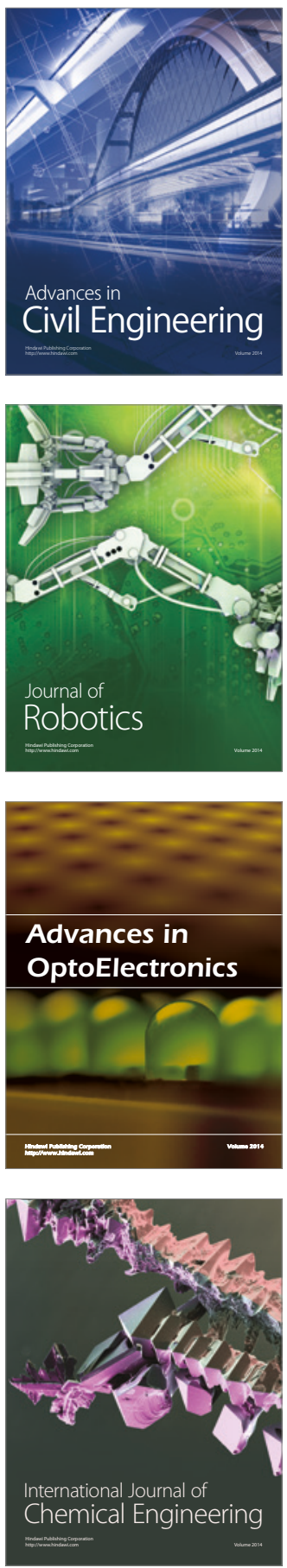

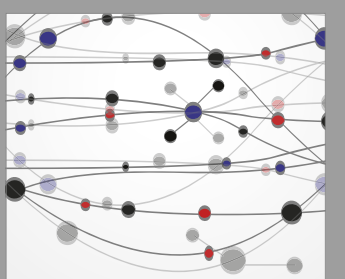

The Scientific World Journal

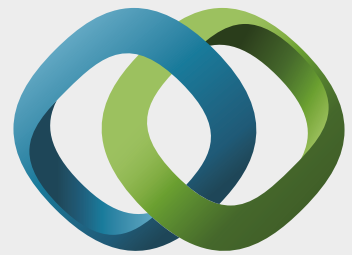

\section{Hindawi}

Submit your manuscripts at

https://www.hindawi.com
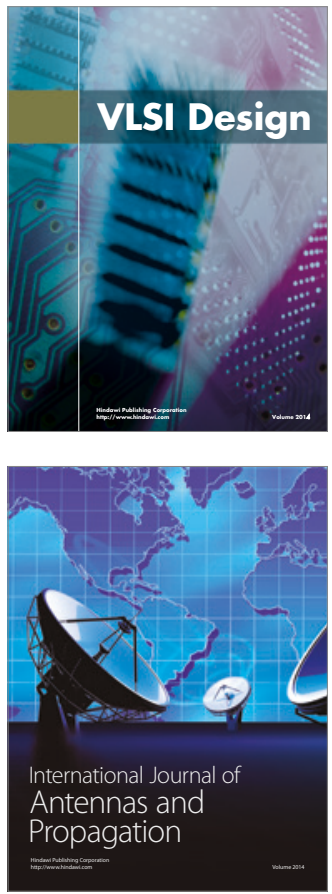

\section{Rotating}

Machinery
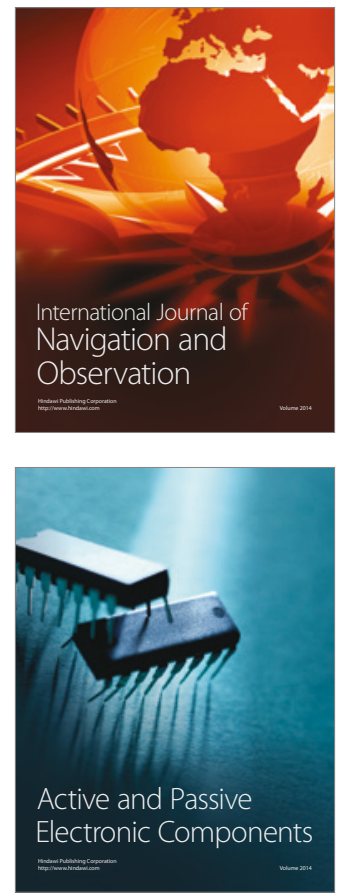
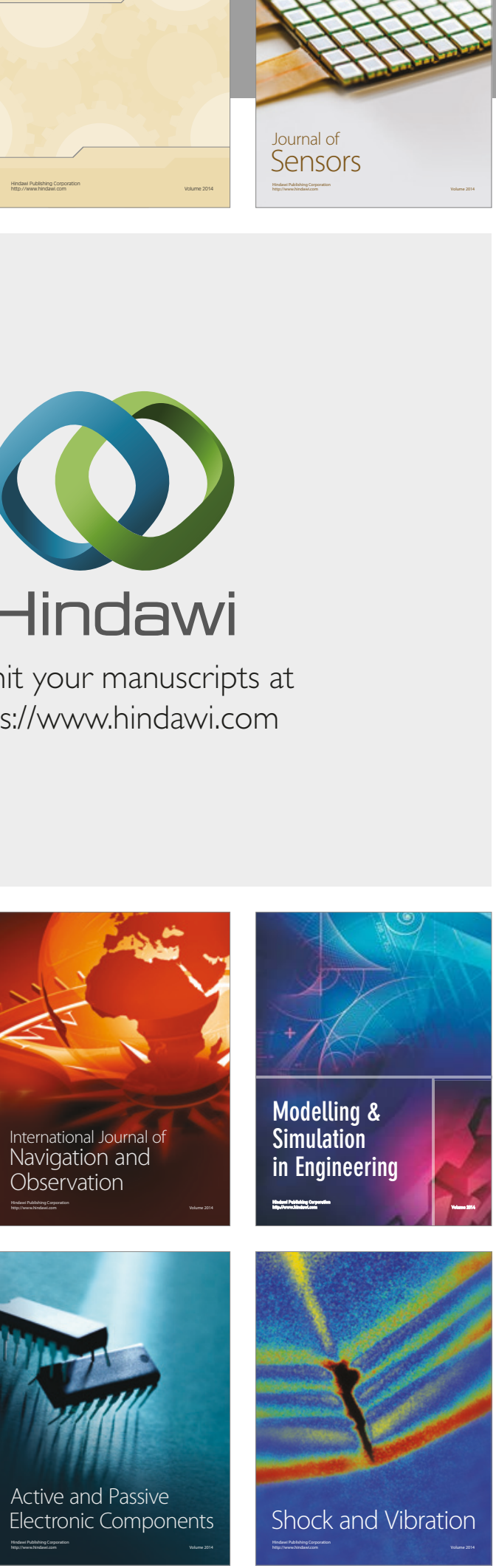
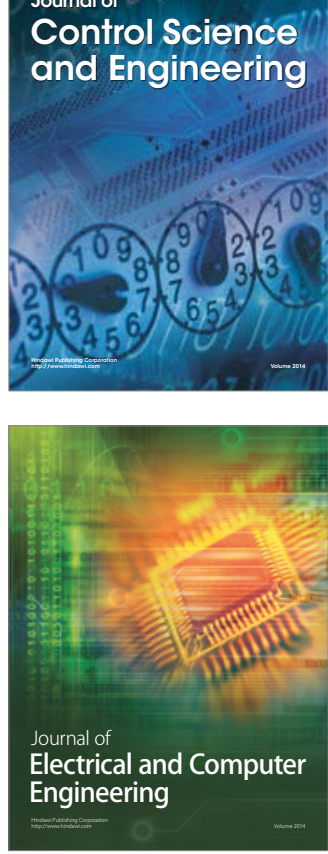

Distributed

Journal of

Control Science

and Engineering
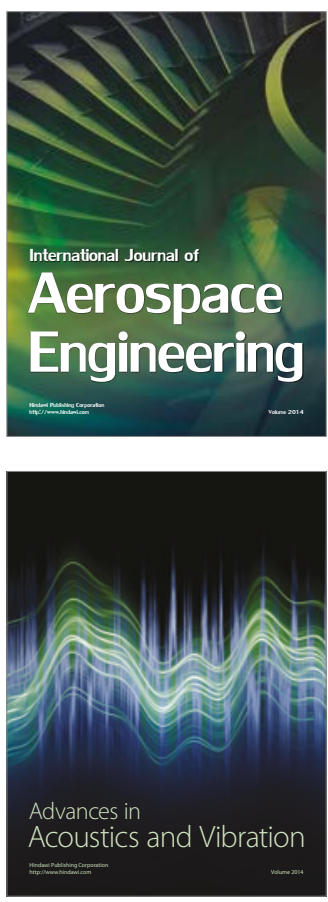

Sensor Networks 